\title{
Analysis of Internal Force Effect in Parallel Manipulators
}

\author{
Mehmet Arif Adli*, Kiyoshi Nagai*, Keiichiro Miyata* and Hideo Hanafusa*
}

Having closed loop structure, parallel manipulators are able to generate internal forces in the joints between the members. This can be achieved by redundantly actuating the manipulators. Although the internal forces are the vectors of force elements that balance each other and do not create any total force, they change the structural stiffness of the manipulators.

In this study, the effect of internal forces on the stiffness of the parallel manipulators was clarifieg based on the analysis of a six degrees of freedom (d. o.f.) spatial parallel manipulator which is composed of at least three sub-arms each having $3 \mathrm{~d}$. o. f.. In the analysis, the static equilibrium state and corresponding internal forces were determined from joint servo potential. Then the stiffness of the manipulator was determined around this equilibrium state. It was shown that, internal forces cause an additional stiffness which depends on the geometry of the manipulator and the magnitude of the internal force. The results were verified both numerically and experimentally for a 3 d.o.f. planar parallel manipulator which is composed of two sub-arms each having 2 d. o. f..

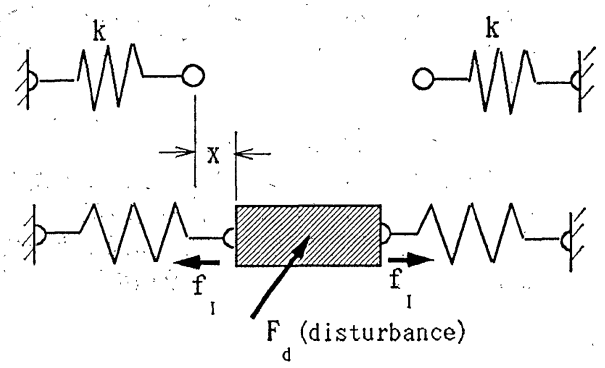

(a) Tensile internal force
Key Words : parallel manipulators, internal forces, stiffness

\section{Introduction}

Parallel manipulators are closed link mechanisms in which the end effector is coupled to a fixed plane through more than one set of drive link trains. Having such a structure, a parallel manipulator consists of redundant joints which are not required to be controlled. In the case of planar parallel manipulators there can be up to three such joints whereas for the spatial parallel manipulators this number reaches to seven depending on the type of the joints used ${ }^{1}$. By actuating some of these joints the compliance at the end effector of the manipulator can be adjusted by controlling each joint compliance independently ${ }^{2)}$. Such a manipulator with redundantly actuated joints gains the ability to generate internal forces in the joints between the members. Since the internal forces are the elements of the force vectors which are balanced in the mechanism, they do not generate any total force. However they influence the structural stiffness of the total mechanism. This can be observed in a simple spring-mass system shown in Fig. 1. It is clear that, when the springs are loaded with tensile internal force, the system is more stiff
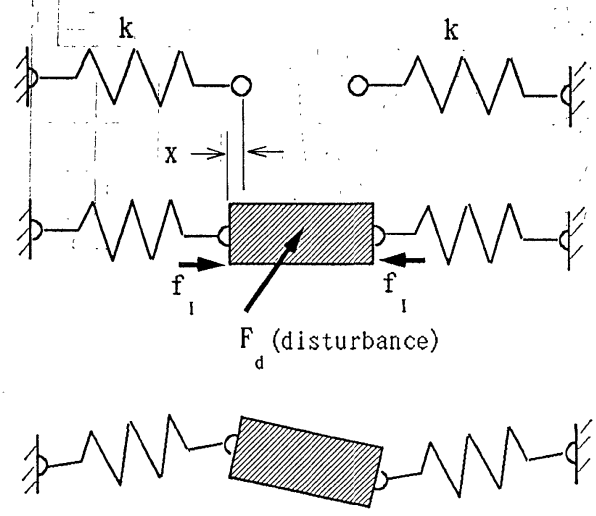

(b) Compressive internal force

Fig. 1 Spring-mass system

\footnotetext{
* Faculty of Science and Engineering, Ritsumeikan University, 56-1 Kitamachi, Tojiin, Kita-ku, Kyoto (Received February 13, 1991)

(Revised May 23, 1991)
} 
to the external disturbances (Fig. 1(a)). Similarly, if the springs are loaded with compressive internal forces, the system will be easily deflected by external disturbances (Fig. 1(b)). This simple example indicates that, the structural stiffness of the mechanisms can be altered by inducing internal forces. A detailed study explaining the principle of the internal force effect on the stiffness of the closed mechanisms can be found in Ref. 3).

Stiffness of the closed link mechanisms has been studied in several works as in Refs. 2) and 4). It is clear that, stiffness is a major property which plays an important role in the overall behavior of a mechanism. Therefore, because of their effect on the stiffness, the existence of internal forces in a closed mechanism is an important factor that must be analyzed. The fact that the stiffness of the closed mechanisms is affected by internal forces means that, by properly. utilizing internal forces the stiffness of a mechanism can be adjusted in some directions of the motion. This is quite useful especially for the cases where the manipulator is required to make fine motions (as in assembly operations) in which the compliance is more important in particular directions.

In this paper, the effect of internal forces on the stiffness of the parallel manipulators around the static equilibrium state was analyzed. The analysis was carried out by considering a general spatial parallel manipulator shown in Fig. 2. The results were verified both numerically and experimentally for a planar parallel manipulator whose end effector has three degrees of freedom of motion.

\section{Effect of the Internal Forces on the Manipulator Stiffness}

\subsection{Static equilibrium state and corresponding internal forces}

Fig. 2(a) shows a general six degrees of freedom spatial parallel manipulator which is composed of an end effector and several drive arms. The necessary notation for an arbitrary $i^{\text {th }}$ arm is given in Fig. $2(\mathrm{~b})$. The manipulator is assumed to be composed of at least three individual arms, each having three degrees of freedom controlled by three actuators. Each actuator is represented by an equivalent spring. When the arms are free, that is at the unconstructed configuration, the springs are unloaded and are at their free lengths. After the arms are combined through an end effector to form a parallel manipulator, this newly formed manipulator reaches a static equilibrium state and generates some amount of internal forces in the joints between each arm and the end effector. The joint displacements at equilibrium state can be found by minimizing the joint servo potential, $P$. The gravitational forces are neglected because they have no essential effect on the result of the analysis.

$$
\mathrm{P}=\frac{1}{2} \sum_{i=1}^{n} \sum_{j=1}^{3} K_{q_{i j}}\left(q_{i j}-q_{u i j}\right)^{2}+\sum_{k=1}^{m} \lambda_{k} \phi_{k}(1)
$$

where

$K_{q_{i j}}:$ stiffness of the $j^{\text {th }}$ joint on the $i^{\text {th }}$ arm

$q_{i j}: j^{\text {th }}$ joint angle on the $i^{\text {th }}$ arm

$q_{u i j}: j^{\text {th }}$ joint angle on the $i^{\text {th }}$ arm of unconstructed (free) configuration

$\phi_{k}: k^{\text {th }}$ constraint equation

$\lambda_{k}: k^{\text {th }}$ Lagrange multiplier

$n$ : number of sub-arms

$m:$ number of constraints

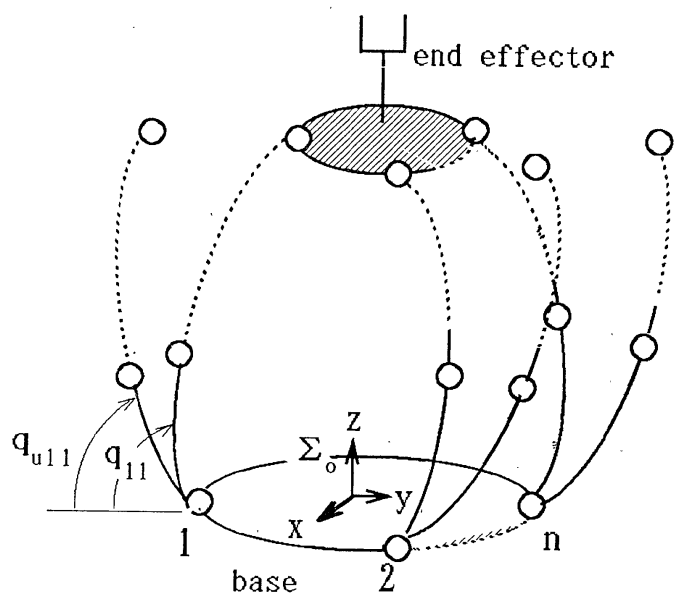

(a)

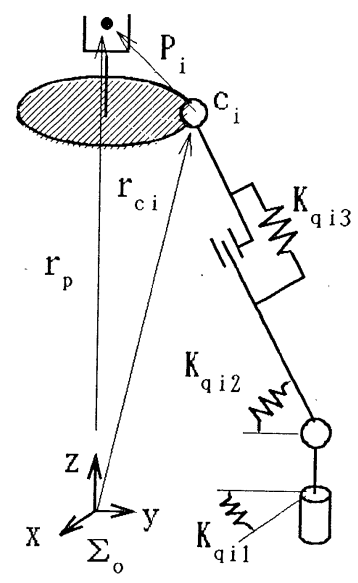

(b)

Fig. 2 Six degrees of freedom spatial parallel manipulator 
Note that the constraint equations $\phi_{k}^{\prime} \mathrm{s}$ are determined from the geometry of the manipulator and,

\section{$\phi_{k}=0 \quad$ for all $k=1, \cdots, m$}

" $\boldsymbol{q}_{e}$ ", the joint displacements at equilibrium state are obtained from

$$
\frac{\partial \mathrm{P}}{\partial \boldsymbol{Q}}=\boldsymbol{O}
$$

where

$$
\begin{aligned}
& \boldsymbol{Q}=\left[\begin{array}{lll}
q_{11}, \cdots, & q_{n 3} & \lambda_{1}, \cdots, \lambda_{m}
\end{array}\right]^{T} \in \mathrm{R}^{3 n+m} \\
& \boldsymbol{q}_{e}=\left[\begin{array}{lll}
\boldsymbol{q}_{e 1}^{T} & \boldsymbol{q}_{e 2}^{T}, \cdots, & \boldsymbol{q}_{e n}^{T}
\end{array}\right]^{T} \\
& \boldsymbol{q}_{e i}=\left[\begin{array}{lll}
q_{e i 1} & q_{e i 2} & q_{e i 3}
\end{array}\right]^{T} \in \mathrm{R}^{3}
\end{aligned}
$$

In the case of any difference between $\boldsymbol{q}_{u i}$ and $\boldsymbol{q}_{e i}$, the joint actuators will be loaded by certain amount of joint torque or force depending on the type of the joint.

The torque or force induced to the joints of each arm at equilibrium state is,

where

$$
\tau_{e i}=\boldsymbol{K}_{q_{i}}\left(\boldsymbol{q}_{e i}-\boldsymbol{q}_{u i}\right)
$$

$$
\begin{aligned}
& \boldsymbol{K}_{q i}=\operatorname{diag}\left[\begin{array}{lll}
K_{q_{i 1}} & K_{q_{i 2}} & K_{q i 3}
\end{array}\right] \in \mathrm{R}^{3 \times 3} \\
& \boldsymbol{q}_{u i}=\left[\begin{array}{lll}
q_{u i 1} & q_{u i 2} & q_{u i 3}
\end{array}\right]^{T} \in \mathrm{R}^{3}
\end{aligned}
$$

Due to this joint load, forces " $\boldsymbol{f}_{\boldsymbol{i}}$ " will be generated at the tip of each arm. At the static equilibrium state, the forces exerted at the tip of the arms balance each other, that is,

$$
\boldsymbol{f}_{1}+\boldsymbol{f}_{2}+\cdots+\boldsymbol{f}_{n}=0
$$

These forces correspond to internal forces $\boldsymbol{f}_{I i}$, which can be determined by

$$
\boldsymbol{f}_{I i}=\left(\boldsymbol{J}_{f 1}^{-1}\right)^{T} \tau_{e i}
$$

$$
\text { where }
$$

$$
\begin{aligned}
\boldsymbol{J}_{f i} & =\frac{\partial \boldsymbol{r}_{c i}}{\partial \boldsymbol{q}_{i}} \in \mathrm{R}^{3 \times 3}: \text { Jacobian of } i^{\text {th }} \text { arm } \\
\boldsymbol{r}_{c i} & =\left[r_{c i x} r_{c i y} r_{c i z}\right]^{T} \\
& \in \mathrm{R}^{3}: \text { position of } i^{\text {th }} \text { arm tip with respect } \\
& \text { to reference frame }
\end{aligned}
$$

Conversely, for the given desired equilibrium state $\left(\boldsymbol{q}_{e i}\right)$ and internal force $\left(\boldsymbol{f}_{I i}\right)$, the joint displacements corresponding to the free state of each arm can be found from

$$
\boldsymbol{q}_{u i}=\boldsymbol{q}_{e i}-\boldsymbol{K}_{q i}^{-1}\left(\boldsymbol{J}_{f i}^{T}\right) \boldsymbol{f}_{I i}
$$

\subsection{Analysis of manipulator stiffness around the equilibrium state}

In previous section, the static equilibrium state of a spatial parallel manipulator and the internal forces acting on the manipulator at the equilibrium state were formulated. In this section, the effect of internal forces on the stiffness of the parallel manipulators will be analyzed. Since the equations involved are highly nonlinear, the analysis is carried out by linearizing the equations around an arbitrary static equilibrium state, denoted by subscript " $e$ ".

For small deflections $\left(\delta \boldsymbol{q}_{i}\right.$ and $\left.\delta \boldsymbol{r}_{c i}\right)$ around the equilibrium state

$$
\boldsymbol{r}_{c i}=\boldsymbol{r}_{c i e}+\delta \boldsymbol{r}_{c i}
$$

$$
\boldsymbol{q}_{i}=\boldsymbol{q}_{e i}+\delta \boldsymbol{q}_{i}
$$

where, $\boldsymbol{r}_{c i}$ is the position of each arm tip with respect to the reference frame and, $\boldsymbol{r}_{c i e}$ is the equilibrium value of $\boldsymbol{r}_{c i}$. For the sake of simplicity, we set $\delta \boldsymbol{r}_{c i}=\boldsymbol{u}_{i}$ and $\delta \boldsymbol{q}_{c i}=\boldsymbol{v}_{i}$. Hence

$$
\frac{\partial}{\partial \boldsymbol{r}_{c i}}=\frac{\partial}{\partial \boldsymbol{u}_{i}} \text { and } \frac{\partial}{\partial \boldsymbol{q}_{i}}=\frac{\partial}{\partial \boldsymbol{v}_{i}}
$$

At the deflected position the potential energy of the $i^{\text {th }}$ arm and the force acting on it are

$$
\begin{aligned}
& \mathrm{P}=\frac{1}{2}\left(\boldsymbol{q}_{i}-\boldsymbol{q}_{u i}\right)^{T} \boldsymbol{K}_{q i}\left(\boldsymbol{q}_{i}-\boldsymbol{q}_{u i}\right) \\
& \boldsymbol{f}_{i}=\frac{\partial \mathrm{P}}{\partial \boldsymbol{r}_{c i}}=\left.\left[\left(\frac{\partial \boldsymbol{q}_{i}}{\partial \boldsymbol{r}_{c i}}\right)^{T} \boldsymbol{K}_{q_{i}}\left(\boldsymbol{q}_{i}-\boldsymbol{q}_{u i}\right)\right]\right|_{\boldsymbol{q}_{e i}+\boldsymbol{v}_{i}}
\end{aligned}
$$

Note that $\frac{\partial \boldsymbol{q}_{i}}{\partial \boldsymbol{r}_{c i}}=\boldsymbol{J}_{f i}^{-1}$ and

$$
\boldsymbol{v}_{i}=\left.{ }_{0}^{\swarrow \boldsymbol{v}_{i}^{\prime}}\right|_{\boldsymbol{q}_{e i}}+\left.\boldsymbol{J}_{f i}^{-1}\right|_{\boldsymbol{q}_{e i}} \boldsymbol{u}_{i}+\left.\frac{\partial^{2} \boldsymbol{q}_{i}}{\partial \boldsymbol{r}_{c i}^{2}}\right|_{\boldsymbol{q}_{e i}} \boldsymbol{u}_{i}^{2}+\cdots
$$

Let $\Delta \boldsymbol{B}_{i}=\left.\frac{\partial^{2} \boldsymbol{q}_{i}}{\partial \boldsymbol{r}_{c i}^{2}}\right|_{\boldsymbol{q}_{e i}} \boldsymbol{u}_{i}^{2}+\cdots$

Substituting Eq. (10) into Eq. (9) yields

$$
\begin{aligned}
\frac{\partial \mathrm{P}}{\partial \boldsymbol{r}_{c i}}= & \left(\boldsymbol{J}_{f i}^{-1}\right)^{T} \mid \boldsymbol{q}_{e i+\boldsymbol{v}_{i}} \boldsymbol{K}_{q_{i}}\left(\boldsymbol{q}_{e i}+\boldsymbol{J}_{f i}^{-1} \mid \boldsymbol{q}_{e i} \boldsymbol{u}_{i}\right. \\
& \left.+\Delta \boldsymbol{B}_{i}-\boldsymbol{q}_{u i}\right)
\end{aligned}
$$

Linearizing $\left(\boldsymbol{J}_{f}^{-1}\right)^{T} \mid \boldsymbol{q}_{e i}+\boldsymbol{v}_{i}$ around $\boldsymbol{q}_{e i}$ by using one of the matrix linearization techniques ${ }^{5)}$, gives

$\left(\boldsymbol{J}_{f i}^{-1}\right)^{T}\left|\boldsymbol{\alpha}_{e i+v_{i}}=\left(\boldsymbol{J}_{f}^{-1}\right)^{T}\right| \boldsymbol{\alpha}_{e i}+\boldsymbol{A}_{i}$

where $\boldsymbol{A}_{\boldsymbol{i}} \in \mathrm{R}^{3 \times 3}$ and its $(j k)^{\text {th }}$ element is

$$
a_{j k}=\left.\left[\boldsymbol{v}_{i}^{T} \frac{\partial s_{j k}}{\partial \boldsymbol{q}_{i}}\right]\right|_{\boldsymbol{q}_{e i}}
$$

or from Eq. (8)

$$
a_{j k}=\left.\left[\boldsymbol{u}_{i}^{T}\left(\boldsymbol{J}_{f}^{-1}\right)^{T} \frac{\partial s_{j k}}{\partial \boldsymbol{q}_{i}}\right]\right|_{\boldsymbol{q}_{e i}}
$$

where $s_{j k}$ is the $(j k)^{\text {th }}$ element of $\left(\boldsymbol{J}_{f}^{-1}\right)^{T}$

substituting Eq. (12) into Eq. (11) yields the linearized form

$$
\frac{\partial \mathrm{P}}{\partial \boldsymbol{r}_{c i}}=\left[\left(\boldsymbol{J}_{f i}^{-1}\right)^{T}+\boldsymbol{A}_{i}\right] \boldsymbol{K}_{q i}\left[\boldsymbol{q}_{e i}-\boldsymbol{q}_{u i}+\boldsymbol{J}_{f i}^{-1} \boldsymbol{u}_{i}+\Delta \boldsymbol{B}_{i}\right]
$$

All $\boldsymbol{J}_{f i}$ matrices in Eq. (14) are constant since they are computed in terms of equilibrium joint angles $\boldsymbol{q}_{e i}$. Differentiating Eq. (14) with respect to $\boldsymbol{r}_{c i}$ again, yields the stiffness at the tip of each arm.

$$
\begin{aligned}
\boldsymbol{K}_{i}=\frac{\partial^{2} \mathrm{P}}{\partial \boldsymbol{r}_{c i}^{2}}= & \left(\boldsymbol{J}_{f i}^{-1}\right)^{T} \boldsymbol{K}_{q_{i}} \boldsymbol{J}_{f i}^{-1}+\frac{\partial}{\partial \boldsymbol{r}_{c i}}\left\{\left(\boldsymbol{J}_{f i}^{-1}\right)^{T} \boldsymbol{K}_{q_{i}} \Delta \boldsymbol{B}_{i}\right\} \\
& +\frac{\partial}{\partial \boldsymbol{r}_{c i}}\left\{\boldsymbol { A } _ { i } \boldsymbol { K } _ { q i } \left[\left(\boldsymbol{q}_{e i}-\boldsymbol{q}_{u i}\right)\right.\right. \\
& \left.\left.+\boldsymbol{J}_{f i}^{-1} \boldsymbol{u}_{i}+\Delta \boldsymbol{B}_{i}\right]\right\}
\end{aligned}
$$

At the static equilibrium state $\boldsymbol{u}_{i} \rightarrow 0$, hence

$$
\begin{aligned}
& \frac{\partial}{\partial \boldsymbol{r}_{c i}}\left\{\left(\boldsymbol{J}_{f i}^{-1}\right)^{T} \boldsymbol{K}_{q_{i}} \Delta \boldsymbol{B}_{i}\right\} \\
& +\frac{\partial}{\partial \boldsymbol{r}_{c i}}\left\{\boldsymbol{A}_{i} \boldsymbol{K}_{q_{i}}\left(\boldsymbol{J}_{f i}^{-1} \boldsymbol{u}_{i}+\Delta \boldsymbol{B}_{i}\right)\right\} \rightarrow 0
\end{aligned}
$$

then, the symmetric arm-tip stiffness matrix $\boldsymbol{K}_{i}$ 
becomes

$$
\boldsymbol{K}_{i}=\boldsymbol{K}_{s i}+\Delta \boldsymbol{K}_{s i}
$$

where $\boldsymbol{K}_{s i} \in \mathrm{R}^{3 \times 3}$ is the stiffness of the joints reflected to the arm tip, and is determined by

$$
\boldsymbol{K}_{s i}=\left(\boldsymbol{J}_{f i}^{-1}\right)^{T} \boldsymbol{K}_{q i} \boldsymbol{J}_{f i}^{-1}
$$

$\Delta \boldsymbol{K}_{s i} \in \mathrm{R}^{3 \times 3}$ is the additional stiffness resulted from the internal force $\boldsymbol{f}_{I i}$ (see Fig. 3 in which $\boldsymbol{K}_{s i}$ and $\Delta \boldsymbol{K}_{s i}$ are represented by virtual springs fixed to the end effector.).

$$
\Delta \boldsymbol{K}_{s i}=\left[\begin{array}{l}
{\left[\frac{\partial \boldsymbol{A}_{i}}{\partial r_{c i x}} \boldsymbol{K}_{q i}\left(\boldsymbol{q}_{e i}-\boldsymbol{q}_{u i}\right)\right]^{T}} \\
{\left[\frac{\partial \boldsymbol{A}_{i}}{\partial r_{c i y}} \boldsymbol{K}_{q_{i}}\left(\boldsymbol{q}_{e i}-\boldsymbol{q}_{u i}\right)\right]^{T}} \\
{\left[\frac{\partial \boldsymbol{A}_{i}}{\partial r_{c i z}} \boldsymbol{K}_{q_{i}}\left(\boldsymbol{q}_{e i}-\boldsymbol{q}_{u i}\right)\right]^{T}}
\end{array}\right]^{T} \in \mathrm{R}^{3 \times 3}
$$

or, substituting Eq. (6), into Eq. (18),

$$
\Delta \boldsymbol{K}_{s i}=\left[\begin{array}{l}
{\left[\frac{\partial \boldsymbol{A}_{i}}{\partial r_{c i x}} \boldsymbol{J}_{f i}^{T_{f i}} \boldsymbol{f}_{I i}\right]^{T}} \\
{\left[\frac{\partial \boldsymbol{A}_{i}}{\partial r_{c i y}} \boldsymbol{J}_{f i}^{T} \boldsymbol{f}_{I i}\right]^{T}} \\
{\left[\frac{\partial \boldsymbol{A}_{i}}{\partial r_{c i z}} \boldsymbol{J}_{f i}^{T} \boldsymbol{f}_{I i}\right]^{T}}
\end{array}\right]^{T} \in \mathrm{R}^{3 \times 3}
$$

Note that $\Delta \boldsymbol{K}_{s i}$ is dependent on both internal

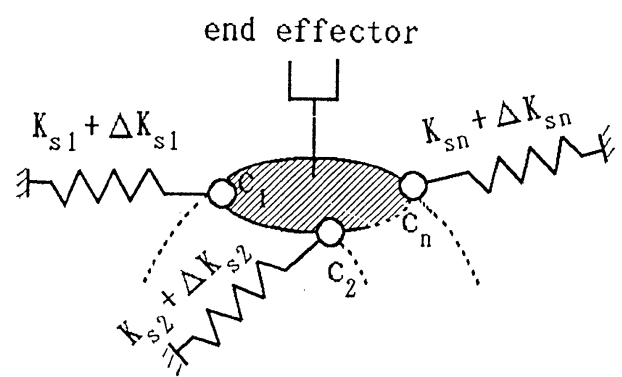

Fig. 3 Stiffness change at the sub-arm tips force $\boldsymbol{f}_{I i}$ and the arm configuration. Once the additional stiffness at the tip of the each arm is determined, the overall change in the end effector stiffness can be calculated by

$$
\Delta \boldsymbol{K}_{e}=\sum_{i=1}^{n} \boldsymbol{J}_{0 i}^{T} \Delta \boldsymbol{K}_{s i} \boldsymbol{J}_{0 i}
$$

where

$$
\begin{aligned}
& \boldsymbol{J}_{0 i}=\frac{\partial\left(\boldsymbol{r}_{p}-\boldsymbol{p}_{i}\right)}{\partial \boldsymbol{r}} \in \mathrm{R}^{3 \times 6} \\
& \boldsymbol{r}=\left[\begin{array}{ll}
\boldsymbol{r}_{p}^{T} & \Theta^{T}
\end{array}\right]^{T} \in \mathrm{R}^{6} \\
& \boldsymbol{r}_{p}=\left[\begin{array}{lll}
r_{p x} & r_{p y} & r_{p z}
\end{array}\right]^{T} \\
& \in \mathrm{R}^{3} \text { : position of manipulator end effector } \\
& \boldsymbol{\Theta}=\left[\begin{array}{lll}
\theta_{1} & \theta_{2} & \theta_{3}
\end{array}\right]^{T} \\
& \in \mathrm{R}^{3} \text { : orientation of manipulator end effector }
\end{aligned}
$$

Then, the total stiffness of the end effector is,

$$
\boldsymbol{K}_{e}=\sum_{i=1}^{n} \boldsymbol{J}_{i}^{T} \boldsymbol{K}_{q_{i}} \boldsymbol{J}_{i}+\Delta \boldsymbol{K}_{e}
$$

where

$$
\begin{aligned}
& \boldsymbol{K}_{e} \in \mathrm{R}^{6 \times 6} \\
& \boldsymbol{J}_{\boldsymbol{i}}=\boldsymbol{J}_{f_{i}}^{-1} \boldsymbol{J}_{0 i}
\end{aligned}
$$

\section{Application to a Three Degrees of Freedom Planar Parallel Manipulator}

In section 2.2 , by analyzing internal force-stiffness relation of a spatial parallel manipulator at static equilibrium state, we have shown that the internal forces alter the structural stiffness of the parallel manipulators. Here in this section we will consider about a planar parallel manipulator shown in Fig. 4. The end effector of this manipulator has three degrees of freedom. The manipulator has six joints. Only three of these joints are required to be actuated in order to determine the position and orientation of the end effector uniquely. The remaining three joints are redundant. In order to generate internal forces in the

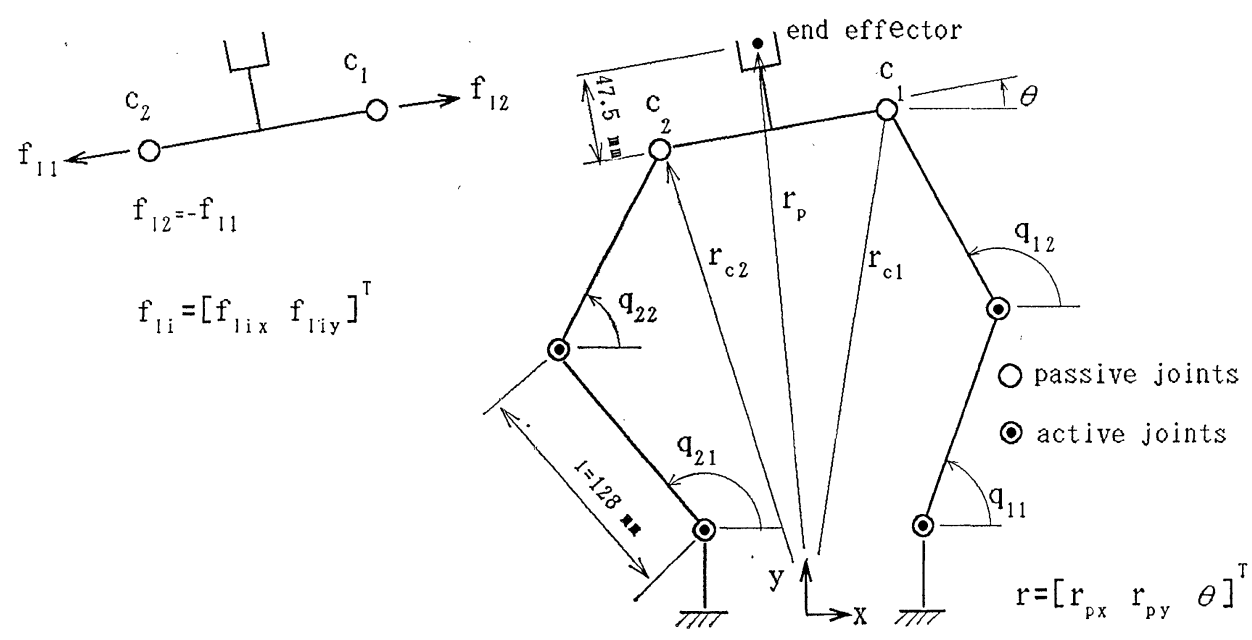

Fig. 4 Three degrees of freedom planar parallel manipulator 
joints between the links at least one of these redundant joints must be actuated. Hence the manipulator is considered to have four actuators. For simplicity, the links are set to equal lengths, "l".

For this parallel manipulator the additional stiffness at the arm tips $c_{1}$ and $c_{2}$ due to internal force, that is the Eq. (19), is

where

$$
\Delta \boldsymbol{K}_{s i}=\left[\begin{array}{ll}
\Delta K_{s i x x} & \Delta K_{s i x y} \\
\Delta K_{s i y_{x}} & \Delta K_{s i y y}
\end{array}\right] \in \mathrm{R}^{2 \times 2}
$$$$
\begin{aligned}
& \Delta K_{s i x x}=\left\{\left[c^{2} q_{i 2} c\left(q_{i 1}-q_{i 2}\right)+c^{2} q_{i 1}\right] s q_{i 1}\right. \\
&\left.-\left[c^{2} q_{i 1} c\left(q_{i 1}-q_{i 2}\right)+c^{2} q_{i 2}\right] s q_{i 2}\right\} \\
& \cdot \frac{f_{I i x}}{l s^{3}\left(q_{i 1}-q_{i 2}\right)} \\
&+\left\{-\left[c^{2} q_{i 2} c\left(q_{i 1}-q_{i 2}\right)+c^{2} q_{i 1}\right] c q_{i 1}\right. \\
&\left.+\left[c^{2} q_{i 1} c\left(q_{i 1}-q_{i 2}\right)+c^{2} q_{i 2}\right] c q_{i 2}\right\} \\
& \cdot \frac{f_{I i y}}{l s^{3}\left(q_{i 1}-q_{i 2}\right)} \\
& \Delta K_{s i x y}=\left\{\left[s q_{i 2} c q_{i 2} c\left(q_{i 1}-q_{i 2}\right)+s q_{i 1} c q_{i 1}\right] s q_{i 1}\right. \\
&\left.-\left[s q_{i 1} c q_{i 1} c\left(q_{i 1}-q_{i 2}\right)+s q_{i 2} c q_{i 2}\right] s q_{i 2}\right\} \\
& \cdot \frac{f_{I i x}}{l s^{3}\left(q_{i 1}-q_{i 2}\right)} \\
&+\left\{-\left[s q_{i 2} c q_{i 2} c\left(q_{i 1}-q_{i 2}\right)+s q_{i 1} c q_{i 1}\right] c q_{i 1}\right. \\
&\left.+\left[s q_{i 1} c q_{i 1} c\left(q_{i 1}-q_{i 2}\right)+s q_{i 2} c q_{i 2}\right] c q_{i 2}\right\} \\
& \cdot \frac{f_{I i y}}{l s^{3}\left(q_{i 1}-q_{i 2}\right)} \\
& \Delta K_{s i x y}= \Delta K_{s i y x} \\
& \Delta K_{s i y y}=\left\{\left[s^{2} q_{i 2} c\left(q_{i 1}-q_{i 2}\right)+s^{2} q_{i 1}\right] s q_{i 1}\right. \\
&+ {\left.\left[s^{2} q_{i 1} c\left(q_{i 1}-q_{i 2}\right)+s^{2} q_{i 2}\right] s q_{i 2}\right\} } \\
& \cdot \frac{f_{I i x}}{l s^{3}\left(q_{i 1}-q_{i 2}\right)} \\
&+\left\{-\left[s^{2} q_{i 2} c\left(q_{i 1}-q_{i 2}\right)+s^{2} q_{i 1}\right] s q_{i 1}\right. \\
&+ {\left.\left[s^{2} q_{i 1} c\left(q_{i 1}-q_{i 2}\right)+s^{2} q_{i 2}\right] c q_{i 2}\right\} } \\
& \cdot \frac{f_{I i y}}{l s^{3}\left(q_{i 1}-q_{i 2}\right)} \\
&
\end{aligned}
$$

here

$$
\begin{aligned}
& s q_{i j}=\sin \left(q_{i j}\right) \text { and } c q_{i j}=\cos \left(q_{i j}\right) \\
& f_{I 1 x}=f_{I} \cos \theta, \quad f_{I 1 y}=f_{I} \sin \theta
\end{aligned}
$$

$$
f_{I 2 x}=-f_{I 1 x} \text { and } f_{I 2 y}=-f_{I 1 y}
$$

$f_{I}$ : magnitude of the internal force

$l:$ length of the links

$i=1,2$ : number or sub-arm

Note that the additional stiffness $\Delta \boldsymbol{K}_{s i}$ depends on both the magnitude of the internal force and the configuration of the mechanism. Indeed $\Delta \boldsymbol{K}_{s i}$ can be written as,

$$
\Delta \boldsymbol{K}_{s i}=\boldsymbol{m}_{i}\left(\boldsymbol{q}_{i}\right) f_{I i x}+\boldsymbol{n}_{i}\left(\boldsymbol{q}_{i}\right) f_{I i y}
$$

and hence arm tip stiffness $\boldsymbol{K}_{i}$ and end effector stiffness $\boldsymbol{K}_{\boldsymbol{e}}$ become,

$$
\begin{aligned}
\boldsymbol{K}_{i}= & \boldsymbol{K}_{s i}+\boldsymbol{m}_{i}\left(\boldsymbol{q}_{i}\right) f_{I i x}+\boldsymbol{n}_{i}\left(\boldsymbol{q}_{i}\right) f_{I i y} \\
\boldsymbol{K}_{e}= & \sum_{i=1}^{2} \boldsymbol{J}_{i}^{T} \boldsymbol{K}_{q_{i}} \boldsymbol{J}_{i}+\boldsymbol{J}_{0 i}^{T}\left[\boldsymbol{m}_{i}\left(\boldsymbol{q}_{i}\right) f_{I i x}\right. \\
& \left.+\boldsymbol{n}_{i}\left(\boldsymbol{q}_{i}\right) f_{I i y}\right] \boldsymbol{J}_{0 i}
\end{aligned}
$$

If the configuration of the mechanism is fixed, that is $\boldsymbol{m}_{i}$ and $\boldsymbol{n}_{i}$ are constant matrices, then $\boldsymbol{K}_{\boldsymbol{i}}$ and $\boldsymbol{K}_{e}$ change linearly with the internal force. The related numerical results are discussed in the next section.

\section{Numerical Results}

By considering the three degress of freedom planar parallel manipulator shown in Fig. 4, several numerical results concerning the analysis above were obtained. As seen in Eq. (23), the variation in stiffness of each sub-arm depends on both the geometrical configuration of the manipulator and the amount of the internal force exerted in the joints. When the manipulator is at a fixed position and orientation, the additional stiffness $\Delta \boldsymbol{K}_{s i}$ at the arm tips changes linearly with the magnitude of the internal force, $f_{I}$. Fig. 6 shows the relation between the components of the arm tip stiffness $\boldsymbol{K}_{i}$ and the internal force $f_{I}$ for both arms at three basic geometrical configurations given in Fig. 5. Notice that, when the manipulator is at a symmetric configuration, both $K_{i x x}$ and $K_{i y y}$ are constant, which means that $\Delta K_{\text {sixx }}=0$ and
$(0,368.5,0)$

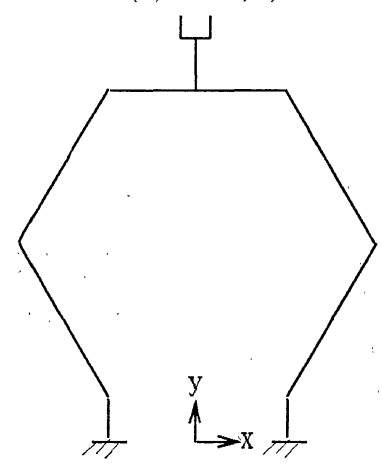

( a )

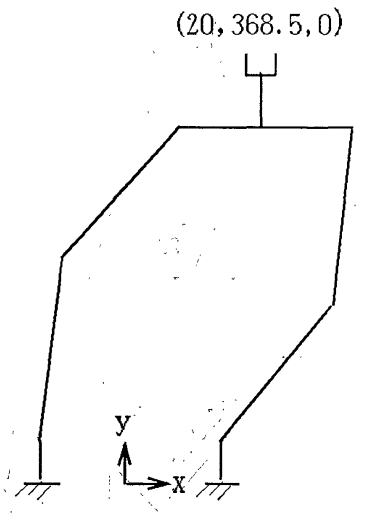

(b)
$(0,368.5,20)$

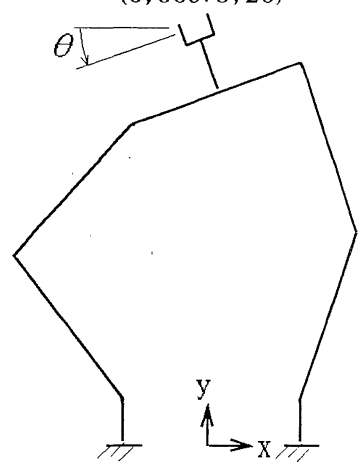

(c)

Fig. 5 Manipulator at different configurations 

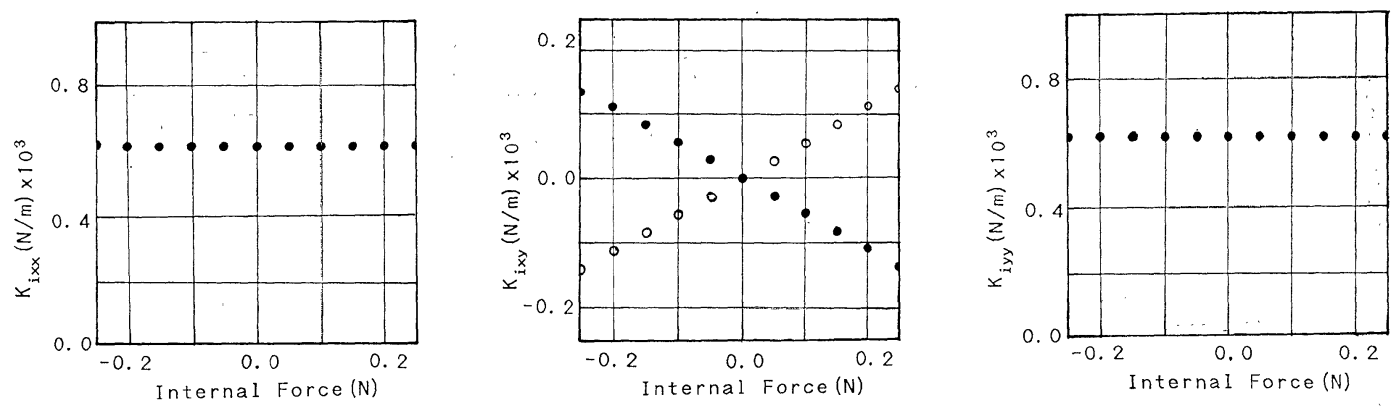

- sub-arm 1 - sub-arm 2

(a) Corresponds to Fig. 5(a)
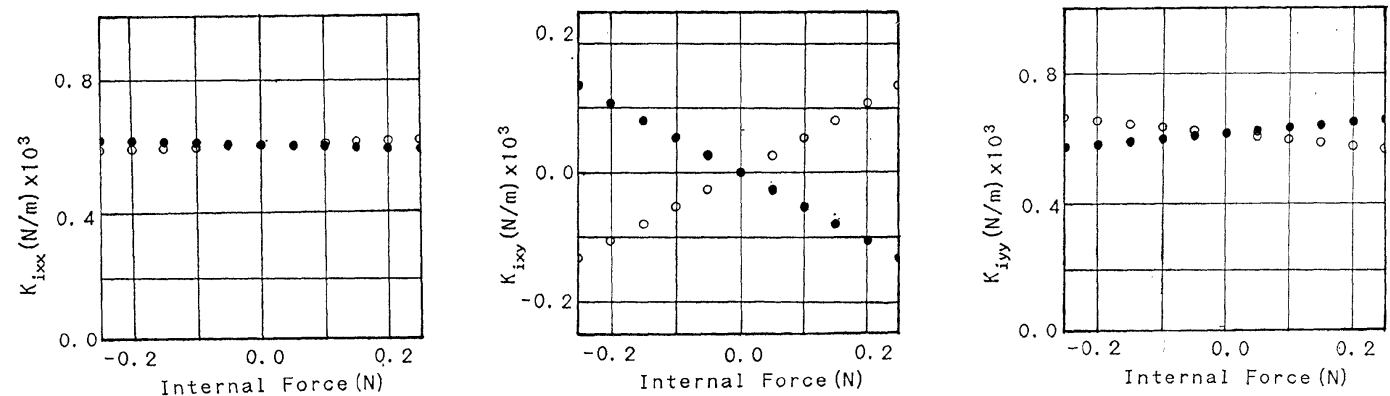

o sub-arm 1 - sub-arm 2

(b) Corresponds to Fig. 5(b)
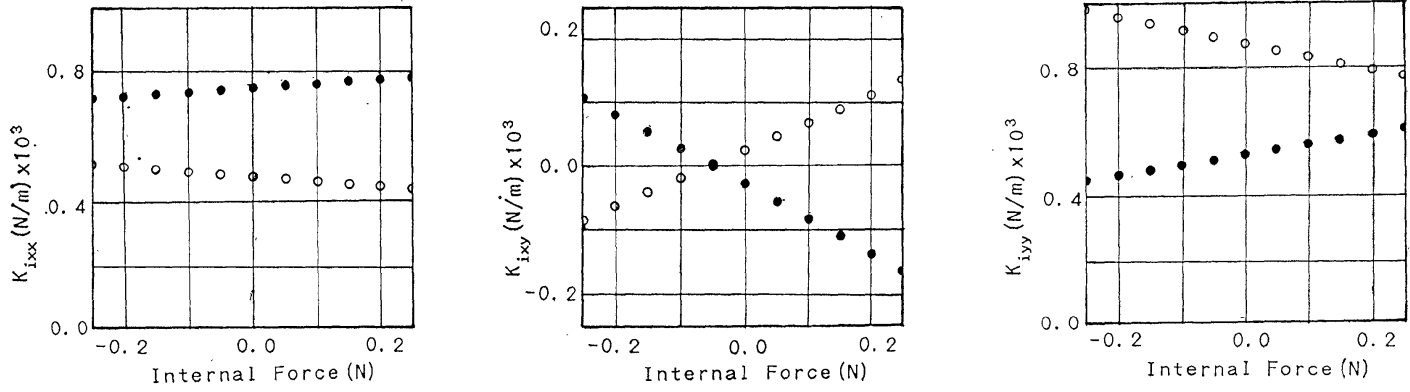

o sub-arm 1 - sub-arm 2

(c) Corresponds to Fig. 5(c)

Fig. 6 Elements of sub-arm tip stiffness matrix, $K_{i}$
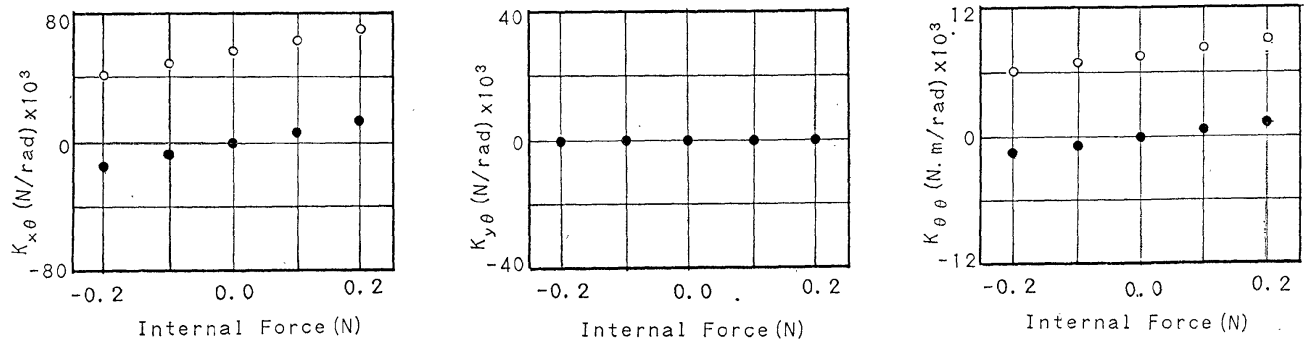

○ $K_{\text {eij }}$ : total stiffness

- $\Delta \mathrm{K}_{\mathrm{eij}}^{\mathrm{ej}}$ :additional stiffness due to the internal force

Fig. 7 Elements of the stiffness matrix at the end effector tip 

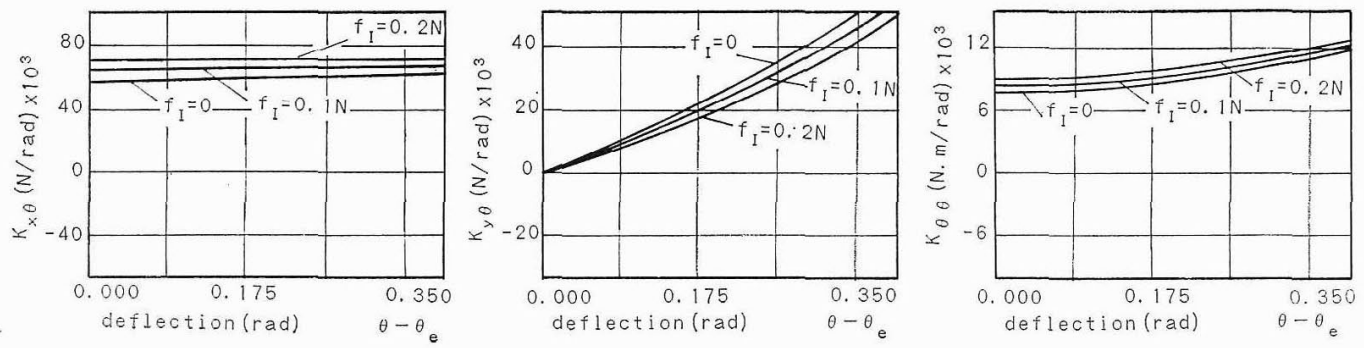

Fig. 8 Variation in the elements of the stiffness matrix at the end effector tip (numerical results)

$\Delta K_{s i y y}=0$. Also, one can see from Fig. 6 (c) that, the variation in $\boldsymbol{K}_{i}$ is strongly dependent to the orientation $(\theta)$ of the manipulator.

The overall stiffness at the end effector of the manipulator is determined by Eq. (25). In Fig. 7, the variation in three components of the end effector stiffness matrix related to the orientation are shown for the special geometrical configuration given in Fig. 5 (a)

The numerical results shown in Figs. 6 and 7 are just for the equilibrium state. In order to see the variation of the stiffness around the equilibrium state, we kept the internal force constant and plot the elements of the stiffness matrix while changing the position and orientation of the end effector. Fig. 8 gives the variation of $K_{x \theta}, K_{y \theta}$ and $K_{\theta \theta}$ of the end effector stiffness matrix while changing only the orientation of the end effector, for three different values of the internal force. The internal forces were defined at the static equilibrium state. As clearly seen from the figure, there is a considerable difference between the stiffness values corresponding to each internal force.

\section{Experiments}

\section{1 Experimental set-up}

The planar parallel manipulator used for the experimental purposes throughout this research was constructed by the combination of two DAIKIN miniature robots as shown in Fig. 9. The geometry of the manipulator is exactly the same as in Fig. 4. The manipulator has three degrees of freedom in terms of two translational and one rotational motion. It is actuated by four DC servo motors in which one of them is redundant.

\section{2 Experimental results}

Fig. 10 shows the variation in the three components of the stiffness matrix defined at the end effector tip, corresponding to three different internal forces. The variation is around the equilibrium state in which the end effector tip was kept at $x=0 \mathrm{~mm}$., $y=368.5 \mathrm{~mm}$, and $\theta=0 \mathrm{rad}$. Similar to the case of numerical results, the internal forces were defined at the equilibrium state. The joint servo gains were set to $K_{q}=0.1 \mathrm{~N} \cdot \mathrm{m} / \mathrm{rad}$. for all the four active joints. In the figure, the horizontal axis represents the rotational deflection of the end effector from the equilibrium state and the vertical

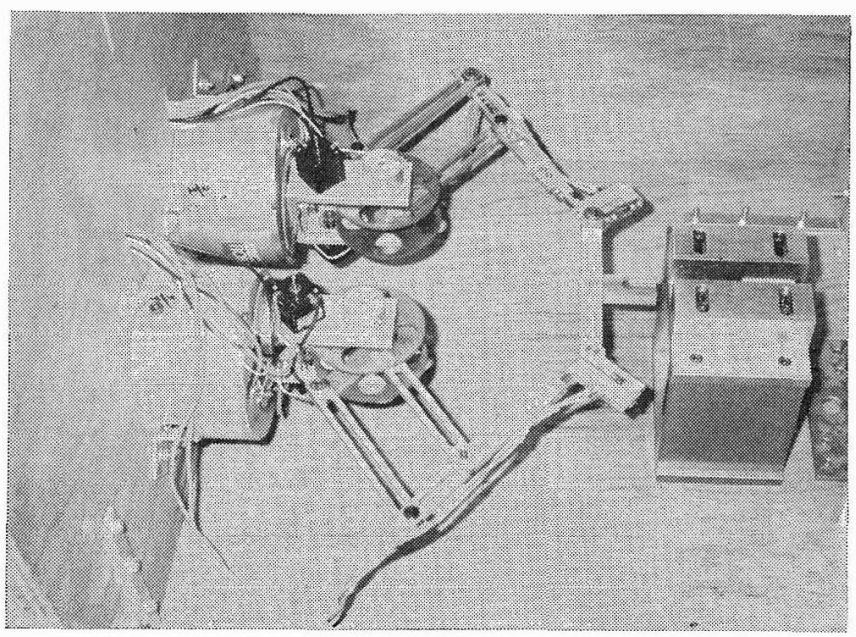

Fig. 9 Planar parallel manipulator 

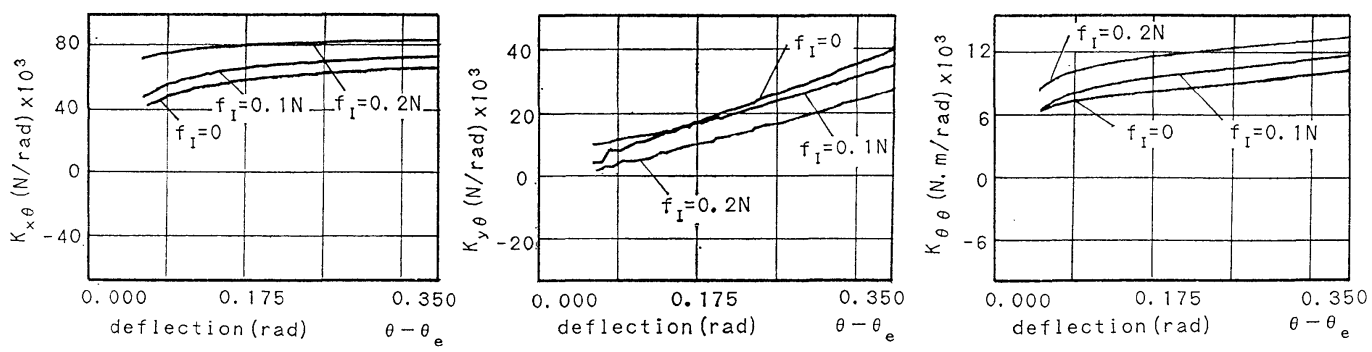

Fig. 10 Variation in the elements of the stiffness matrix at the end effector tip (experimental results)

axis represents the variation in the specified component of the end effector stiffness matrix. The end effector was kept at a fixed position and only its orientation was changed. Therefore the measured stiffness components refer to the rotational components of the stiffness matrix. The experiment was repeated for three different internal forces. Here, only the mostly varied three components are shown. Due to the noise in the signals received from the measuring devices, it was not possible to measure the stiffness at very near to the equilibrium state. However by extending the curves in Fig. 10, the stiffness at the vicinity of the equilibrium can be approximately detected. Similar to the numerical results (see Fig. 8), it is clear that for each internal force the stiffness has different value which indicates that the structural stiffness of the manipulator is affected by the internal forces.

\section{Conclusion}

Effect of internal forces on the structural stiffness of the parallel manipulators was analyzed. The theoretical analysis was carried out by considering a redundantly actuated six degrees of freedom spatial parallel manipulator which can generate internal forces in the joints. The static equilibrium state and corresponding internal forces in the manipulator were determined from the joint servo potential. The stiffness of each sub-arm tip and manipulator end effector were analytically determined around the static equilibrium state. It was shown that, the structural stiffness at each subarm tip and hence the stiffness of the manipulator end effector is changed by the existence of the internal forces in the manipulator. The effect of internal forces depends on the magnitude of the internal force as well as the geometrical configuration of the manipulator. The analysis was applied to a three degrees of freedom planar parallel manipulator, and the results were confirmed both numerically and experimentally, using this manipulator.

The effect of internal forces will be useful in assembly operations in which the compliance is more important. Furthermore, there are some situations where links compose the closed mechanism such as grasping by multi-finger hands. The effect of internal forces should be considered in such problems as stable prehension.

\section{References}

1) Y. Nakamura and M. Ghodoussi : A Computational Scheme of Closed Link Robot Dynamics Derived by D'Alambert Principle, Proceedings of IEEE International Conference on Robotics and Automation, 3, 1354/1360 (1988)

2) K. Yokoi, M. Kaneko and K. Tanie: A Compliance Control Method Suggested by Muscle Networks in Human Arms, Proceedings of IEEE International Workshop on Intelligent Robots and Systems [IROS '88], 385/390, Tokyo (1988)

3) H. Hanafusa and M. A. Adli : Effects of Internal Forces on Stiffness of Closed Mechanisms, 8th. Annual Conference of Robotics Society of Japan, 3, 931/934 (1990) (in Japanese)

4) G. Clement: Stiffness Mapping for Parallel Manipulators, IEEE Trans. Robotics and Automation, RA-6-3, 371/382 (1990)

5) W. J. Palm III : Modeling, Analysis, and Control of Dynamic Systems, John Wiley and Sons (1983) 